\title{
Phasorial DPP-BOTDA sensor tolerant to non-local effects
}

\author{
Javier Urricelqui, Jon Mariñelarena, Mikel Sagues, Alayn Loayssa* \\ Universidad Pública de Navarra, Campus Arrosadia 31006, Spain
}

\begin{abstract}
We introduce a modification of the differential pulse-width pair technique in a BOTDA sensor based on a phase-modulated probe wave and RF demodulation. This provides a differential Brillouin phasorial signal with high spatial and spectral resolution in both components (magnitude and phase-shift). Moreover, the use of a phase-modulated probe wave provides RF phase-shift measurements tolerant to the emergence of non-local effects. The combination of both techniques can lead to the development of long-range BOTDA sensors. Proof-of-concept experiments demonstrate $\mathrm{RF}$ phase-shift measurements with $1 \mathrm{~m}$ spatial resolution over $50 \mathrm{~km}$ and an uncertainty of $1.3^{\circ} \mathrm{C}$ at the worst contrast position.
\end{abstract}

Keywords: Phasorial BOTDA, non-local effects, spatial resolution, RF demodulation.

\section{INTRODUCTION}

Brillouin optical time domain analysis (BOTDA) sensors provide unique capabilities to realize distributed measurements of temperature and strain over conventional single mode fibers (SMF). They are considered an attractive technology for several industrial sectors due to its potential to monitor large areas of coverage with high spatial resolution ${ }^{1}$. However, as the demand for larger distance range steadily increases, the limitations from so-called non-local effects become more serious $^{2}$. The latter is caused by the continuous energy transferred between the probe and pump wave along the fiber, which modifies the pump power by introducing a wavelength dependence that leads to errors in the Brillouin frequency shift (BFS) measurements. This is a major concern for which several countermeasures have been proposed ${ }^{2,3,4}$.

Another key challenge is the spatial resolution of the measurements, which is directly related to the pump pulse length. However, decreasing the temporal duration of the pump pulses leads to a broadening of the measured Brillouin spectrum that reduces the measurement precision. There are different proposals to overcome this limitation ${ }^{5,6}$. Among them, the differential pulse-width pair (DPP) technique ${ }^{6}$ is one of the most convenient in long-range BOTDA sensors due to its effectiveness and easy deployment.

Recently, we have demonstrated a novel technique that increases the tolerance of BOTDA sensors to non-local effects ${ }^{7}$. It is based on a phase-modulated probe wave and RF demodulation that provides the advantage of RF phase-shift measurements that are largely immune to variations of the pump pulse power. In this work, we introduce a modification of the DPP pair technique that takes advantage of the complex signals (magnitude and phase-shift) supplied by our system. Instead of just subtracting the amplitude of the BOTDA signal for two pulses with different duration, it is based on a full phasor subtraction of both responses. This leads to a differential Brillouin phasor signal that merges the advantages of both techniques: tolerance to non-local effects and high spatial resolution using the RF phase-shift signal.

\section{FUNDAMENTALS OF THE SYSTEM}

Stimulated Brillouin scattering (SBS) is the non-linear optical effect that governs the operation of BOTDA sensors. It is based on the narrowband amplification of a probe wave within an optical fiber when a pump signal is introduced at the opposite end of a SMF. Distributed measurements are performed by pulsing the pump wave and measuring the probe wave as a function of the time-of-flight of the pump wave at the fiber. It is known that the spatial resolution in conventional BOTDA sensors is given by the spatial coverage of the pulse-width equivalent fiber length; hence it increases when shorter pulses are deployed. However, when the pulse duration is of the order of the acoustic-phonon lifetime (around $10 \mathrm{~ns} / 1 \mathrm{~m}$ ), it broadens the measured Brillouin line-width giving rise to uncertainties at the temperature or strain measurements ${ }^{6}$.

*alayn.loayssa@unavarra.es; phone 0034 948169840; fax 0034 948169720; unavarra.es 
In order to avoid this trade-off between spatial resolution and the BFS precision, the DPP technique has been widely deployed in long-range BOTDA sensors. It is based on the subtraction of two time-domain Brillouin signals sequentially measured by injecting two pump pulses with different pulse duration ${ }^{6}$. As a consequence, the differential Brillouin signal has much higher spatial resolution due to the removal of the common interaction between both pulses and the measured differential Brillouin gain spectrum presents a narrow line-width because of the long duration pulses.

In contrast to the conventional DPP-BOTDA sensor that only measure the magnitude, we deploy this technique but using the RF signal (magnitude and phase-shift) detected by employing a phase-modulated probe wave and RF demodulation ${ }^{7}$. The detected phasor corresponding to a particular position of the fiber is given by ${ }^{7}$ :

$$
I(z) \approx \frac{4 R_{D} E_{0} E_{S B} g_{0} \Delta v_{B}}{\sqrt{\Delta v_{B}^{2}+(2 \Delta v)^{2}}} \exp \left(-j \arctan \left(2 \frac{\Delta v}{\Delta v_{B}}\right)\right) .
$$

where $E_{0}$ and $E_{S B}$ are the amplitudes of the optical fields of the carrier and first sidebands of the phase-modulated probe, $R_{D}$ is the responsivity of the photodetector, $g_{0}$ is the local Brillouin peak gain, $\Delta v_{B}$ is the Brillouin line-width, and $\Delta v$ is the detuning of the interacting sideband from the center of the Brillouin spectrum at that particular location. As Eq. 2 shows, the RF phase-shift signal has no dependence with the particular local Brillouin gain associated to SBS process. As a consequence, it does not depend on the pump power. This has the major implication that RF phase-shift based measurements are not going to be affected by non-local effects because they are independent of the wavelength-dependent changes of the pump pulse power ${ }^{7}$.

In the proposed technique, each pump pulse injected into the fiber provides a complex time-domain trace (magnitude and phase-shift). The differential signal is given by the phasor subtraction of both components at each location of the fiber. This process is schematically depicted in Fig. 1, where a fiber section with a given BFS $\left(\mathrm{BFS}_{1}\right)$ has a short strained section $\Delta u\left(\mathrm{BFS}_{2}\right)$. The resultant RF signal at a particular location of the first fiber section is affected by the integrated Brillouin gain and phase-shift experienced by the probe wave over the length covered by the first pulse introduced into the fiber, yielding the corresponding phasor. Injecting a second pump pulse of differential duration $(\Delta u)$, the RF signal measured for the same location will be affected by the complex Brillouin spectra of both fiber sections inducing a change in the amplitude and phase-shift of the second phasor. As it is shown, the differential RF signal will be given by the subtraction of both phasors, this yields a differential phasor whose RF phase-shift is largely immune to non-local effects. This RF phase-shift, $\Delta \theta_{R F}$, corresponds to the strained fiber section; hence enhanced spatial resolution is obtained.
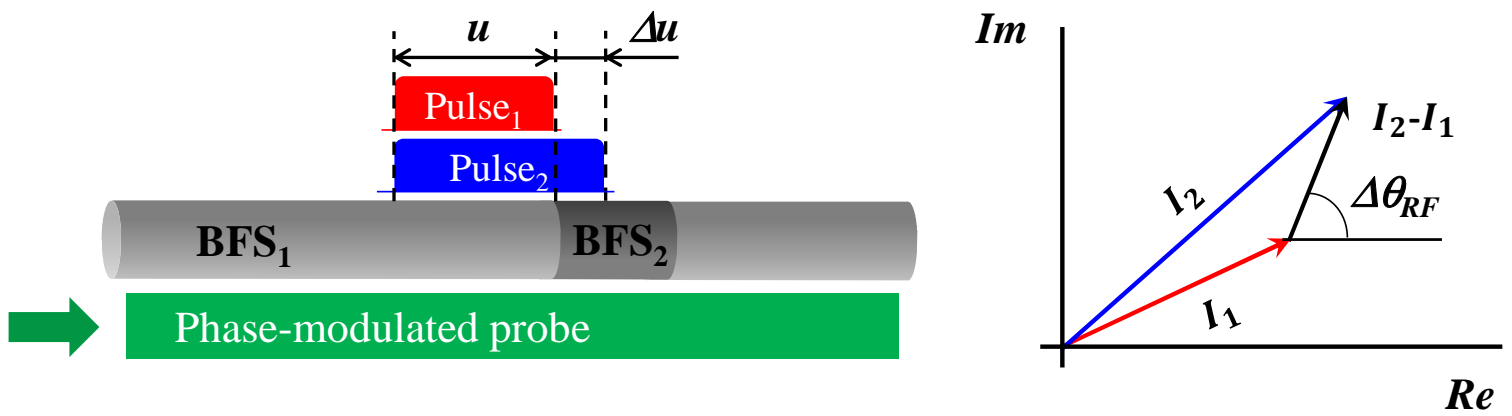

Figure 1. Sketch of DPP technique using a BOTDA sensor based on a phase-modulated probe and RF demodulation.

\section{EXPERIMENTAL RESULTS}

The experimental setup depicted in Fig. 2 was assembled in order to evaluate the potential of the technique. The light from a distributed feedback (DFB) laser diode at $1560 \mathrm{~nm}$ is coupled into two optical branches. In the upper branch, a double-sideband suppressed-carrier modulation is generated with a Mach-Zehnder electro-optic modulator (MZ-EOM). The resultant signal is pulsed using a semiconductor optical amplifier (SOA) and then amplified by an erbium-doped fiber amplifier (EDFA). The upper-sideband of the pulsed signal is selected using a narrowband fiber Bragg grating (FBG) obtaining the desired pump wave and then a polarization scrambler (PS) is used. In the lower branch, a probe wave is generated with an electro-optic phase modulator driven by a $1.3-\mathrm{GHz} \mathrm{RF}$ signal. Once the probe wave has interacted with the pump pulse via SBS is directed to a dispersion compensating module (DCM), which compensates most of the dispersion introduced by the $50 \mathrm{~km}$-long SMF sensing fiber. Finally, the probe signal is detected and the resultant RF signal is demodulated and captured in a digital oscilloscope. 


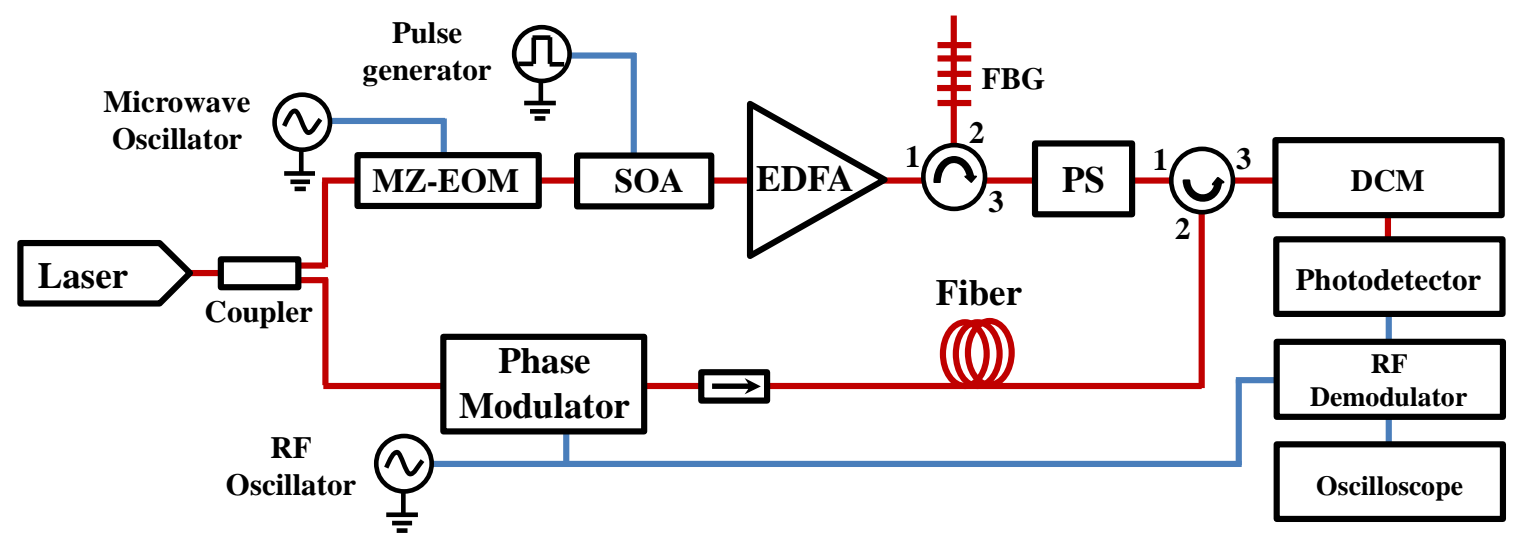

Figure 2. Experimental setup for the DPP-BOTDA sensor based on phase modulated probe wave and RF demodulation.

In order to induce non-local effects at the system, a probe with $0.54 \mathrm{~mW}$ of power is injected into the fiber. It results in amplification of the pump pulse power (this is a loss-based BOTDA sensor configuration) due to the accumulated energy transfer from the probe wave to the pump wave during its propagation. Figure 3 shows this effect, where the pump power for each frequency difference between probe and pump waves is represented. Note that a $115 \%$ of pump amplification is obtained when the frequency difference of both waves matches the average BFS of the optical fiber (set as $\Delta v=0 \mathrm{MHz}$ ). This value far exceeds the maximum tolerable factor ${ }^{2}(17 \%)$, which induces a measurement error of $1 \mathrm{MHz}$. This frequency dependence of the pump signal distorts the measured Brillouin gain spectrum when the temperature of a spot located at the probe end of the fiber, is modified ${ }^{2}$. This was demonstrated by inserting a $13 \mathrm{~m}$-length section at the end of the fiber in a climatic chamber at $61^{\circ} \mathrm{C}(\Delta v=30 \mathrm{MHz})$. Fig. 3 depicts the measured amplitude and RF phase-shift spectra for that section using $50 \mathrm{~ns}$ pump pulses. Two measurements were actually performed, swapping the fiber inputs for the two interacting waves, which serves to check non-local effects ${ }^{2}$. Notice that when the hot-spot is located at the probe end of the fiber, the amplitude spectrum is distorted and there is a biasing effect that introduces a measurement error. This is due to the frequency dependence that is acquired by the pump pulse in its journey along the fiber. In contrast to the amplitude spectra, the shape of the RF phase-shift spectra remains unaltered for both measurements.

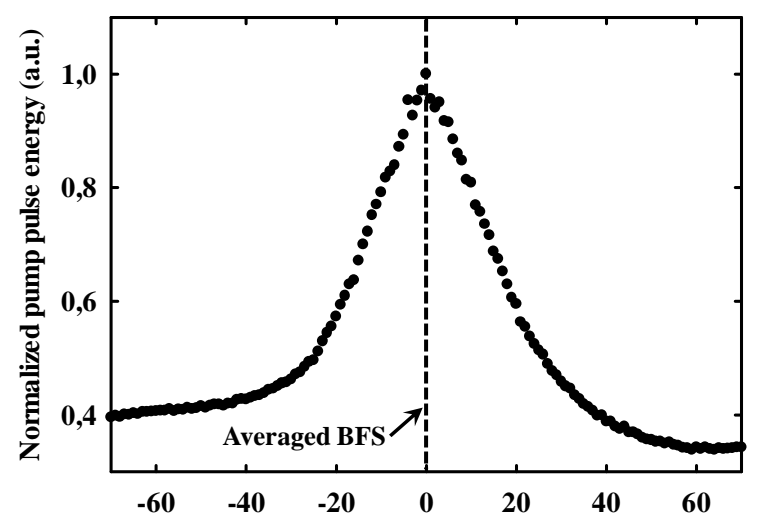

(a)

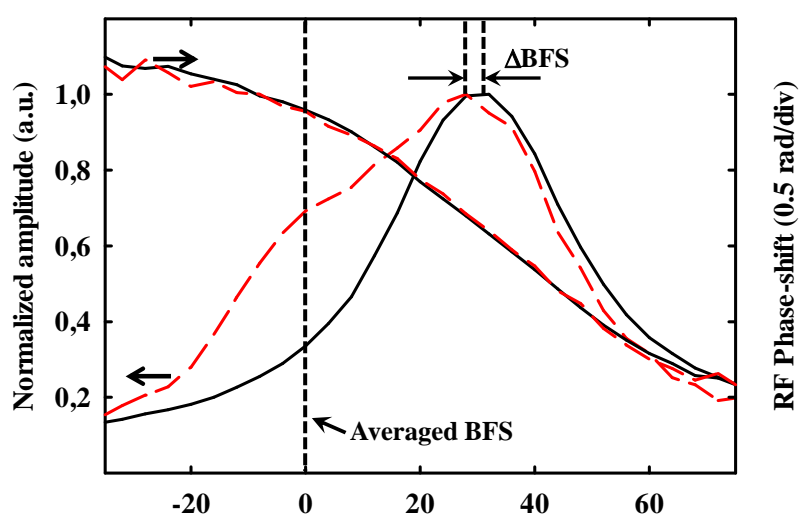

(b)

$\Delta v(\mathbf{M H z})$

Figure 3. (a) Measured pump pulse energy at the end of the fiber. (b) Measured amplitude and RF phase-shift spectra at the hot spot section when that section is located at the probe input (red dashed line) or at the pump input (black solid line).

A similar experiment was performed but, this time, deploying the modified DPP technique, i.e. sequentially injecting two pump pulses of 50 and $60 \mathrm{~ns}$ and vector subtracting the time-domain BOTDA traces. The experimental results shown in Fig. 4, match those obtained using $50 \mathrm{~ns}$ pulses, apart from the expected reduction of SNR due to the enhanced spatial resolution. The precision achieved in the BFS measurements was $1.3 \mathrm{MHz}\left(1.3^{\circ} \mathrm{C} / 26 \mu \varepsilon\right)$ at the worst contrast position. Finally, one meter of fiber was extracted from the final section placed at the climatic chamber and placed at room temperature in a loose state in order to demonstrate the spatial resolution enhancement. Fig. 4 depicts the color-coded RF phase-shift distribution along the fiber final section. At the location corresponding to the room-temperature meter section, a shift from $\Delta v \sim 30 \mathrm{MHz}$ to $\sim-5 \mathrm{MHz}$, which fits the temperature change for that section, is clearly appreciable. 

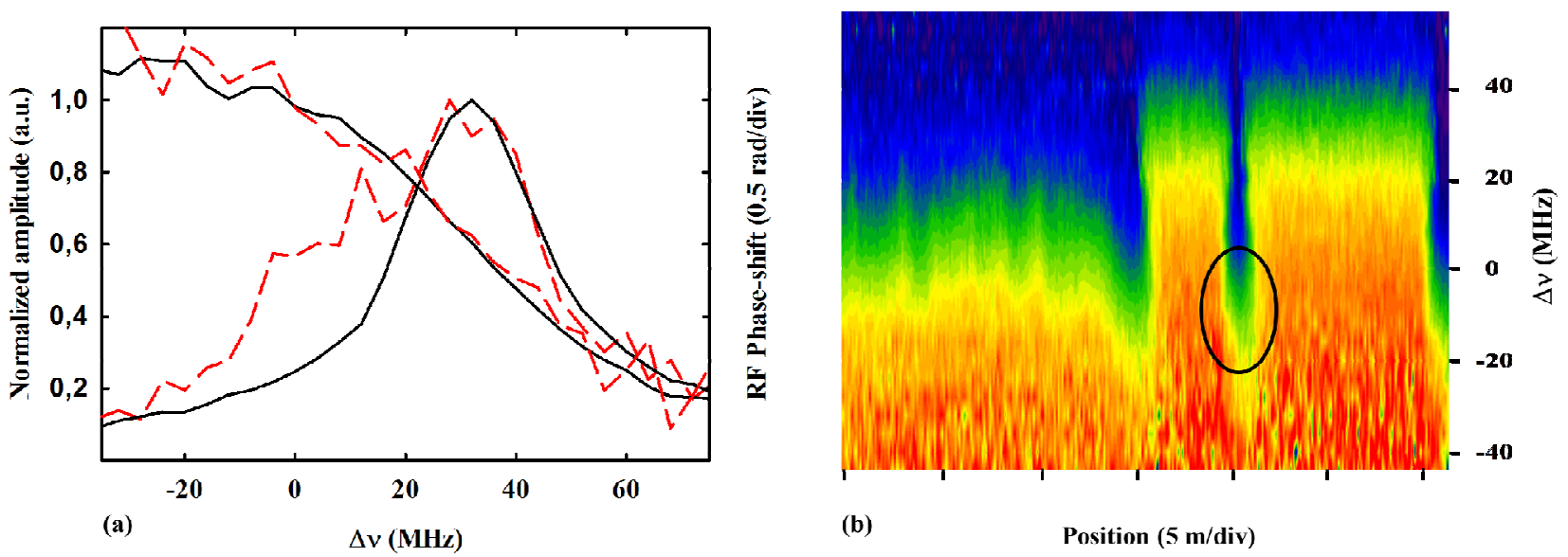

Figure 4. (a) Differential amplitude and RF phase-shift spectra at the hot spot section when that section is located at the probe input (red dashed line) or at the pump input (black solid line). (b) Differential RF phase-shift distribution.

\section{CONCLUSION}

In this work we have presented a long-range BOTDA sensor that merges the advantages of the DPP technique and the deployment of a phase-modulated probe wave and RF demodulation. The former provides RF phase-shift measurements with high spatial and spectral resolution and the latter gives rises to RF phase-shift spectra that have no dependence with the apparition of non-local effects. Therefore, two of the fundamental drawbacks of the conventional long-range BOTDA are overcome. Finally, an experimental proof-of-concept has been performed with a $50 \mathrm{~km}$ long fiber obtaining unaltered phase-shift spectra in presence of non-local effects. Moreover, a $1 \mathrm{~m}$ spot has been detected by using the RF phase-shift of the differential Brillouin vector signal.

\section{ACKNOWLEDGMENTS}

The authors wish to acknowledge the financial support from the Spanish Ministerio de Ciencia e Innovación through the project TEC2010-20224-C02-01 and from the Universidad Pública de Navarra.

\section{REFERENCES}

[1] Angulo-Vinuesa, X., Martin, S., Corredera, P. and Gonzalez-Herraez, M., "Raman-assisted Brillouin optical time-domain analysis with sub-meter resolution over $100 \mathrm{~km}$," Opt. Express 20(11), 12147-12154 (2012).

[2] Thévenaz, L., Foaleng Mafang, S. and Lin, J., "Effect of pulse depletion in a Brillouin optical time-domain analysis system," Opt. Express 21(12), 14017-14035 (2013).

[3] Dong, Y., Chen, L. and Bao, X. "System optimization of a long-range Brillouin-loss-based distributed fiber sensor," Appl. Opt. 49(27), 5020-5025 (2010).

[4] Minardo, A., Bernini, R., Zeni, L., Thévenaz, L. and Briffod, F., "A reconstruction technique for long-range stimulated Brillouin scattering distributed fibre-optic sensors: Experimental results, " Meas. Sci. Technol. 16(4), 900-908 (2005).

[5] Song, K. Y., Chin, S., Primerov, N. and Thévenaz, L., "Time-domain distributed fiber sensor with $1 \mathrm{~cm}$ spatial resolution based on Brillouin dynamic grating," J. Lightw. Technol. 28(14), 2062-2067 (2010).

[6] Li, W., Bao, X., Li, Y. and Chen, L., "Differential pulse-width pair BOTDA for high spatial resolution sensing," Opt. Express 16(26), 21616-21625 (2008).

[7] Urricelqui, J., Sagues, M. and Loayssa, A., "BOTDA measurements tolerant to non-local effects by using a phase-modulated probe wave and RF demodulation," Opt. Express 21(14), 17186-17194 (2013). 
Copyright 2014 Society of Photo Optical Instrumentation Engineers. One print or electronic copy may be made for personal use only. Systematic re-production and distribution, duplication of any material in this paper for a fee or for commercial purposes, or modification of the content of the paper are prohibited. 